\title{
EDITORIAL
}

\section{Diversity, Health, and the State of Patient Care in the US Health Care System}

\author{
Hayden B. Bosworth, $P h D^{1,2,3}$, and Ronnie D. Horner, PhD

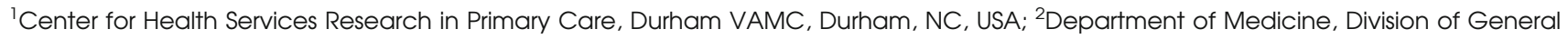 \\ Internal Medicine, Duke University Medical Center, Durham, NC, USA; ${ }^{3}$ Department of Psychiatry and Behavioral Sciences, School of Nursing, \\ Duke University, Durham, NC, USA; ${ }^{4}$ Department of Public Health Sciences, College of Medicine, University of Cincinnati, Cincinnati, OH, USA.
}

J Gen Intern Med 24(Suppl 3):567

DOI: $10.1007 / \mathrm{s} 11606-009-1124-4$

(C) Society of General Internal Medicine 2009

$\mathrm{T}$ he current supplement adds to a growing body of literature by examining factors related to quality of health care with an emphasis on patient-centered care in the context of the Latino population. Despite being the largest of the racial/ethnic groups within the US, we have only begun to explore Latino perspectives on health and health care and the ways in which these perspectives influence their utilization of health care. It should be noted that we refer to perspectives rather than perspective. This is deliberate. The Latino population is a heterogeneous population comprised of numerous ethnicities that are related, but distinct. Indeed, the racial and ethnic categories that we use in health research belie the heterogeneity of cultures within those categories, including the typical referent-non-Hispanic white. Patient-centered care, then, offers an approach by which the US health care system can begin to accommodate this extensive diversity among individual patients.

Rodriguez et al. ${ }^{1}$ in their introduction to this supplement define patient-centeredness as providing care that is respectful of and responsive to individual preferences, needs and values, ensuring that patient preferences inform clinical decisions. Quality patient-centered care necessitates that clinicians do a good job of assessing patient needs, expectations and environment, using information from patients about their family, cultural background, health and health care behaviors, primary language, health care literacy and other factors to improve and design new services. The articles in this supplement begin to provide evidence on the needs, expectations and environment of Latino patients.

Related, there is an effort to 'transform' or 're-engineer' the delivery of primary care in the United States. One way to transform primary care is the use of the 'medical home' model (initially suggested in 1960s in the pediatric field). Medicare and other large health care insurers explored the 'medical home' as a way to improve quality, satisfaction and outcomes for patients with chronic medical illnesses. The Joint Principles of the Patient-Centered Medical Home consists of individuals having a personal physician who provides first contact care that is continuous, comprehensive and accessible, while being coordinated with the care offered by other providers ${ }^{2}$. Thus, the core features of medical home include a physician-directed medical practice; a personal doctor for every patient; the capacity to coordinate high-quality, accessible care; and payments that recognize a medical home's added value for patients ${ }^{3}$. Such strategies require an evidence base to understand which components should be included to maximize benefit, yet limit cost. Clearly, an understanding of the patient's culture will be central to effective implementation of the "medical home" concept.

This supplement includes a series of articles with information about experiences of Latinos using the United States health care systems. In so doing, we believe the aim of this supplement has been met: to provide new knowledge and advance the conceptual and operational grounding for future policy-relevant research relating to Latino health. As we move forward with addressing issues related to patient-centered care and reconceptualizing how medical care is provided in the primary care, there is a need to test ways to not only implement quality of care, but to also determine how to sustain these efforts. This latter goal will surely require significant thought regarding reimbursement and financial incentives. Given the potential of the current health care system in the US, there is hope that we can move away from small incremental steps, truly think 'outside the box,' and implement and evaluate patient-centered programs that result in significant health care improvements for all Americans that are sustainable. All of this requires knowledge of not only the patient's health status, but his or her cultural heritage that influences that status. The type of inquiry being reported in this supplement on the Latino cultures and health is a model or template for lines of investigation that need to be undertaken for the other diverse populations that comprise these United States.

Corresponding Author: Hayden B. Bosworth, PhD; Department of Medicine, Department of Psychiatry and Behavioral Sciences, School of Nursing, Duke University Medical Center, HSRD (152), Suite 600, 411 West Chapel Hill Street, Durham, NC 27701, USA (e-mail: hayden.bosworth@duke.edu).

\section{REFERENCES}

1. Rodriguez M. Introduction to the Latino supplement. J Gen Intern Med. 2009.

2. American College of Physicians. Joint principles of the patient-centered medical home. http://www.acponline.org/advocacy/where_we_stand/ medical_home/approve_jp.pdf. Accessed January 29, 2009.

3. Iglehart JK. No place like home-testing a new model of care delivery. N Engl J Med. 2008;359(12):1200-202. 